\title{
Relación de los cultivos modificados genéticamente con el ambiente y la salud de la población costarricense
}

\author{
Ana M. Espinoza ${ }^{1,2}$, Griselda Arrieta-Espinoza ${ }^{1} \&$ Ana Sittenfeld $^{1}$ \\ 1 Centro de Investigación en Biología Celular y Molecular, Universidad de Costa Rica, Ciudad Universitaria Rodrigo \\ Facio, San José, Costa Rica; amespino@racsa.co.cr. \\ 2 Escuela de Fitotecnia, Facultad de Agronomía, Universidad de Costa Rica, Ciudad Universitaria Rodrigo Facio, San \\ José, Costa Rica.
}

Recibido 15-VII-2003. Corregido 02-IX-2003. Aceptado 02-IX-2004.

\begin{abstract}
Relationship of genetically modified crops with the environment and health of the Costa Rican human population. Genetic engineering and the food derived from genetically modified crops (GMCs) have been the center of debate worldwide, as has occurred historically with the advent of new technologies. Questions are derived from the potential impact of GMCs to the environment and the safety of the products to the consumers. In relation to the first inquiry, practice has been oriented to a case-by-case-study, according to the own characteristics of the GMC, in order to minimize its impact in the environment. Scientific studies in diverse latitudes of the world have demonstrated that GMCs in the market showed no adverse effects related to this issue. In relation to food derived from the GMCs, rigorous evaluation protocols have been developed and approved by FAO and WHO to guarantee the innocuousness of these products. Up to the moment, no contraindications for human health have been pointed out for the products that are available today in the market. In the particular case of Costa Rica, the country has established since the 90 s a regulatory biosafety framework for the management of the GMCs, safeguarding the biodiversity of the country and the health of consumers. At the same time the country has made significant public and private investments in the field that allowed the country to obtain a leading position in biosafety in the region and genetic engineering research at national research centers. Any attempt to restrict or prohibit these activities in the country, will put in risk the previously described investment, will affect the generation of new knowledge for decision making and the leadership in the field, preventing the benefits derived from this promising technology. Rev. Biol. Trop. 52(3): 727-732. Epub 2004 Dic 15.
\end{abstract}

Key words: transgenics, GMCs, Costa Rica, biosafety, regulatory framework.

Palabras clave: transgénicos, OGMs, Costa Rica, bioseguridad, marco regulatorio.

Durante las próximas décadas, la producción de alimentos requerirá de un incremento significativo para responder a los rápidos cambios, tales como el crecimiento en la población mundial, el aumento en la competencia internacional, la globalización y la mayor demanda por parte de los consumidores de alimentos de mejor calidad, más seguros y más nutritivos (Bourlag 2000, Huang et al. 2002).

Como es bien sabido las variedades vegetales obtenidas por mejoramiento genético tradicional tuvieron un impacto importante durante la Revolución Verde (1950-1985). Sin embargo, estas variedades han disminuido recientemente su rendimiento, además de ser muy demandantes en términos de fertilizantes, pesticidas y agua. Por lo tanto, para las próximas décadas se deben producir nuevas variedades para responder a la creciente demanda de alimentos de mejor calidad nutricional pero seguros para la salud y el ambiente (Conway y Toenniessen 1999).

La ingeniería genética es una de las tecnologías llamadas a satisfacer las necesidades antes apuntadas con beneficios para Costa Rica como un todo y para los productores y 
consumidores. Beneficiaría al país ya que permite la producción de cultivos con mejores rendimientos, más eficientes en el uso del agua y del suelo, resistentes a factores adversos como la sequía, plagas y enfermedades, además de incorporar mejoras en la calidad nutritiva (vitaminas y minerales) (Huang et al. 2002).

Esta tecnología involucra la aplicación de técnicas celulares y moleculares, que permiten la incorporación en los cultivos de caracteres útiles (genes) provenientes de diversas fuentes (bacterias, plantas y animales entre otros) y que pueden complementar los procesos de mejora genética tradicional y las prácticas de agricultura sostenible (BIO 2002). A estos cultivos se les ha denominado como: cultivos genéticamente modificados (CGM), transgénicos, recombinantes o productos biotecnológicos.

Si hacemos un poco de historia, cabe recordar que la primera generación de CGMs llegó al mercado internacional en 1996 con la siembra de 1 millón de ha de soya en los Estados Unidos. Desde entonces, la adopción de los CGMs se ha incrementado en forma sostenida durante 7 años. En el 2003 se sembraron en el mundo 67.7 millones de ha que representan un $20 \%$ del total de la producción mundial de maíz, soya, algodón y canola. Además, estos se producen en 18 países por 7 millones de productores, de los cuales el $85 \%$ son pequeños agricultores de países en vías de desarrollo (James 2003, BIO 2002). La amplia utilización de soya, maíz, algodón y canola modificados genéticamente radica en que aportan beneficios reales para los agricultores tales como aumentos en la producción, reducción en los costos de insumos, disminución del uso de insecticidas, mejor control de insectos y malezas de una forma amigable con el ambiente, mejor conservación de los suelos y aumento en el retorno económico (Huang et al. 2002, Qaim y Zilberman 2003). Asimismo, los CGMs han traído importantes beneficios para los consumidores. Por ejemplo, el maíz-Bt ha reducido en un $93 \%$ la contaminación del grano con micotoxinas, potentes carcinógenos, asimismo, su utilización ha disminuido significativamente la contaminación con residuos tóxicos de insecticidas sintéticos que se utilizaban en el control de insectos (NCFAP 2002).

Pese a todo lo anterior, la ingeniería genética y los alimentos derivados de los CGMs ha sido objeto de debate a escala mundial. Históricamente se ha visto que el surgimiento de tecnologías novedosas produce inquietudes en la población quien se cuestiona, en este caso, si los CGMs son seguros al ambiente y si los productos derivados de ellos son inocuos para los consumidores. Se estima que los alimentos desarrollados por métodos tradicionales y que se han ingerido por cientos de años son seguros, a pesar de que algunas de sus características agronómicas y nutricionales se alteraron durante el proceso de mejoramiento. Sin embargo, los alimentos tradicionales no han requerido la evaluación de riesgo para su consumo.

En el caso de los alimentos derivados de los CGMs el panorama es muy diferente, ya que se han desarrollado sistemas de evaluación rigurosa para permitir su consumo y comercialización (Hails 2002). Actualmente hay protocolos experimentales estandarizados y aprobados por la FAO y la OMS para determinar la inocuidad de los productos derivados de los CGMs (Kuiper et al. 2001). Esta inocuidad se establece mediante el concepto de equivalencia sustancial, que consiste en demostrar la equidad entre un alimento derivado del CGM y el original antes de la modificación genética (Kuiper 2003). Para ello se evalúan los posibles efectos del nuevo alimento en la salud como toxicidad, alergenicidad, contenido nutricional y cualquier efecto inesperado resultado de la modificación genética (Haslberger 2003). Los alimentos biotecnológicos disponibles en el mercado en otros países han pasado por una estricta evaluación y en ningún caso se han detectado contraindicaciones para la salud humana (Kuiper et al. 2001, ICSU 2003). En forma adicional, el consumo de estos alimentos en su análisis de caso por caso, no ha producido ningún efecto nocivo en las poblaciones que los consumen (FAO y WHO 2000, WHO 2003). 
A la fecha las autoridades costarricenses no han recibido solicitudes para la importación de alimentos derivados de CGMs, pero se deberían acreditar desde ahora laboratorios locales para que en el futuro realicen las pruebas de equivalencia sustancial, de acuerdo con los protocolos internacionales.

La bioseguridad ambiental de los CGMs es particularmente importante para Costa Rica debido a su gran biodiversidad y recursos fitogenéticos (Sittenfeld et al. 2001). El fenómeno de flujo de genes que ocurre en la naturaleza y que origina nuevos tipos de plantas es en parte responsable de esta gran diversidad. Debido a que la ingeniería genética permite la utilización de genes de diferentes fuentes, existe la inquietud de que el flujo de genes desde los CGMs pueda afectar la biodiversidad ya que las especies silvestres podrían recibir genes de especies relacionadas. No obstante, para que ocurra intercambio genético entre plantas, deben compartir el hábitat, coincidir en la floración, ser genéticamente compatibles y la característica transferida debe conferir a la planta receptora una ventaja adaptativa (Burke y Rieseberg 2003, Conner et al. 2003). Si la característica introducida no confiere ventajas adaptativas, se perdería en unas cuantas generaciones y no tendría efecto sobre la población de plantas receptoras (Ellstrand et al. 1999, Nuffield Council on Bioethics 2004).

Los estudios sobre el flujo de genes adquieren mayor relevancia en centros de origen de los cultivos donde generalmente existen especies silvestres relacionadas (tal sería el caso del Teosintle en México, especie silvestre de donde se domesticó el maíz). Por estas razones, previo a la liberación de un CGM deben realizarse estudios caso-por-caso con la finalidad de desarrollar estrategias para minimizar el flujo de genes, si el mismo fuese detectado. En Costa Rica, actualmente se llevan a cabo estudios de flujo de genes de arroz a especies silvestres relacionadas (Quesada et al. 2002, Arrieta et al. 2002, Zamora et al. 2003, Arrieta-Espinoza et al. 2004). Es importante indicar que ya existen estrategias moleculares que permiten introducir los genes en el genoma del cloroplasto de las plantas y así reducir significativamente las posibilidades de que el gene introducido se disperse por medio del polen (Daniell 1999, Nuttfield Council on Bioethics 2004).

En materia de control, la Comisión Técnica Nacional de Bioseguridad (CTNB) creada en 1990 y adscrita al Ministerio de Agricultura y Ganadería (Ley de Protección Fitosanitaria de Costa Rica 7664) tiene como objetivo elaborar las normas, mecanismos y medidas para garantizar la seguridad para la salud y el ambiente en la investigación, desarrollo, producción, aplicación, liberación e introducción de organismos modificados por medio de ingeniería genética. Mediante sus actividades la CTNB salvaguarda el derecho constitucional a tener un ambiente sano y ecológicamente equilibrado (Arjona 2004). Actualmente, la CTNB no ha aprobado la siembra de CGMs destinados al consumo local humano o animal, solamente se ha autorizado siembras para el incremento de semilla de CGMs como maíz, soya y algodón con resistencia a plagas, enfermedades y malezas, semilla que se reexporta en su totalidad a los países de origen. Asimismo, la Universidad de Costa Rica, el CATIE y empresas privadas locales han recibido autorización de la CTNB para realizar investigaciones confinadas con cultivos como arroz, maíz, banano y plátano, con el propósito de conferirles resistencia a enfermedades y a herbicidas (Valdez et al. 2004a, Valdez et al. 2004b Valdez et al. 2004c).

Los beneficios derivados de la experiencia con los CGMs en el país propiciaron la organización de un marco regulatorio en bioseguridad. Asimismo, se fortaleció la investigación local, la capacitación de funcionarios y científicos de instituciones estatales, el desarrollo de empresas nacionales, la contratación de personal calificado y la inversión nacional y extranjera en instalaciones y equipos (MAG 2004). Esto le permitió a Costa Rica, posicionarse en Latinoamérica como uno de los primeros países con fortalezas en el campo de la biotecnología y la bioseguridad. 
Existe también la inquietud en algunos sectores de la sociedad de que la producción de semilla de CGMs tendría algún impacto en la seguridad alimentaria del país, ya que se considera que su comercialización está principalmente en manos de compañías transnacionales. Cabe destacar que para la venta de semilla de CGMs no se han creado nuevas compañias semilleristas y la comercialización la llevan a cabo las mismas empresas que venden semillas convencionales. Por lo tanto, el problema del posible monopolio de semilla no es inherente a la nueva tecnología (Ramón 2004). Además, investigaciones en 76 instituciones públicas de países en vías de desarrollo han producido 46 CGMs, que eventualmente podrían comercializarse por compañias nacionales (Atanassov et al. 2004).

Diferentes gobiernos e instituciones costarricenses han apoyado el desarrollo de la biotecnología agrícola mediante el financiamiento de institutos de investigación, lo que ha llevado al mejoramiento genético de muchos cultivos, en algunos casos como en la Universidad de Costa Rica incluso con tecnologías de punta como la ingeniería genética. El país cuenta con la infraestructura, equipo y personal científico para utilizar la ingeniería genética en el mejoramiento de otros cultivos importantes para Costa Rica y resolver problemas fitosanitarios locales que no son de interés comercial para las empresas transnacionales.

Es importante destacar que las restricciones actuales existentes en Europa en cuanto a la importación de productos derivados de los CGM, tienen implicaciones para la exportación de productos como el banano o el café producidos en Costa Rica. Aún cuando los cultivos no sean transgénicos, la Unión Europea, con sus políticas de trazabilidad y etiquetado para alimentos derivados de los CGMs, podría solicitar que se demuestre que los productos de estos cultivos no son modificados genéticamente o en su defecto realizar ellos mismos las pruebas correspondientes. Esto podría significar una importante barrera no arancelaria para el comercio. Por esta razón, es importante que el país desarrolle la capacidad para responder a esta demanda, mediante la acreditación de laboratorios que certifiquen si se trata o no de un alimento derivado de un CGM.

En resumen, el país ha acumulado ya una sólida experiencia en materia de bioseguridad e investigación científica en el campo de los CGMs, lo que garantiza el control eficiente de esta actividad en forma integral, de acuerdo con los estándares internacionales y ha permitido el desarrollar y acumular conocimiento sobre las consecuencias de los CGMs, en la relación biodiversidad-biotecnología. Por lo tanto, cualquier intento de restricción, o peor aún, de prohibición de dicha actividad, pondría en riesgo la cuantiosa inversión de recursos hecha hasta ahora, afectaría el liderazgo que ostenta Costa Rica en la actualidad, la capacidad de desarrollar nuevo conocimiento para la toma de decisiones y daría al traste con los beneficios económicos, sociales y ambientales de esta prometedora tecnología.

\section{RESUMEN}

La ingeniería genética y los alimentos derivados de los cultivos genéticamente modificados (CGMs) han sido objeto de debate a escala mundial, como ocurre históricamente con el surgimiento de tecnologías novedosas. Se cuestiona si los CGMs son seguros al ambiente y si los productos derivados de ellos son inocuos para los consumidores. Sobre la primera de esas inquietudes, la práctica se ha orientado a estudiar caso por caso, según las propias características del CGM, para minimizar su impacto en el ambiente. Estudios científicos en diversas latitudes han demostrado que no ha habido efectos dañinos en este particular. En cuanto a los alimentos derivados de los CGMs se han desarrollado sistemas de evaluación rigurosa para permitir su consumo y comercialización, y hay protocolos aprobados por la FAO y la OMS para garantizar su inocuidad. Hasta el momento, en ningún caso se han detectado contraindicaciones para la salud humana en los productos disponibles hoy en el mercado. Por su parte, Costa Rica estableció desde los años 90 un marco regulatorio en bioseguridad que se encarga de garantizar la seguridad para la salud y el ambiente en el manejo de los CGMs. Asimismo, se ha hecho una gran inversión estatal y privada en esta área, lo que le permitió a Costa Rica posicionarse en la región como uno de los primeros países con fortalezas en materia de bioseguridad y de investigación en ingeniería genética en centros de investigación nacionales. Cualquier intento de restringir o prohibir dicha actividad pondría en riesgo esa inversión, afectaría la generación de nuevo 
conocimiento para la toma de decisiones y el liderazgo alcanzado en el campo y nos privaría de los beneficios de esta prometedora tecnología.

\section{REFERENCIAS}

Arrieta, G., T. Quesada, E. Gamboa, E. Sánchez \& A.M. Espinoza. 2002. Transgenic rice and gene flow assessment to wild and weedy rice species in Costa Rica. In C.R. Roseland (ed.). Abstracts of the OECD International Conference LMOS and the Environment, held in Raleigh, North Carolina, USA. pp. 69-79.

Arrieta-Espinoza G., E. Sánchez, S. Vargas, J. Lobo, T. Quesada \& A.M. Espinoza. 2004. The weedy rice complex in Costa Rica. I. Morphologic study and relationship with comercial rice varieties and wild Oryza relatives. Genet. Resources Crop Evol.: en prensa.

Atanassov, A., A. Bahieldin, J. Brink, M. Burachik, J.I. Cohen, V. Dhawan, R.V. Ebora, J. Falck-Zepeda, L. Herrera-Estrella, J. Komen, F. C. Low, E. Omaliko, B. Odiambo, H. Quemada, Y. Peng, M.J. Sampaio, I. Sithole-Niang, A. Sittenfeld, M. Smale, R. Valyasevi, Y. Zafar \& P. Zambrano. 2004. To Reach the Poor - Results from the ISNAR-IFPRI Next Harvest Study on Genetically Modified Crops, Public Research, and Policy Implications. EPTD Discussion Paper No. 116. International Food Policy Research Institute IFPRI, Washington D.C. 57 p.

Borlaug, N.E. 2000. Ending world hunger. The promise of biotechnology and the threat of antiscience zealotry. Plant Physiol. 124: 487-490.

Burke, J.M. \& L.H. Rieseberg. 2003. Fitness effects of transgenic disease resistance in sunflowers. Science 300: 1250 .

Conner, A.J., T.R. Glare \& J-P. Nap. 2003. The release of genetically modified crops into the environment. Part II. Overview of ecological risk assessment. Plant J. 33: 19-46.

Conway, G \& G. Toenniessen. 1999. Feeding the world in the twenty-first century. Nature 402: C55-C58.

Daniell, H. 1999. New tools for chloroplast genetic engineering. Nat. Biotech. 17: 855-856.

Ellstrand, N.C., H.C. Prentice \& J.F. Hancock. 1999. Gene flow and introgression from domesticated plants into their wild relatives. Ann. Rev. Ecol. Syst. 30: 539563.

Espinoza, A.M., A. Sittenfeld \& S. Salazar. 2003. Developing transgenic rice at the University of Costa Rica: perspectives and considerations for managing intellectual property rights. Interciencia 28: 111-117.

FAO \& WHO. 2000. Safety aspects of genetically modified foods of plant origin. Report of a joint FAO/WHO expert consultation on foods derived from biotechnology, WHO, Geneva, Switzerland, (Geneva: WHO). 37 p.

Hails, R.S. 2002. Assessing the risks associated with new agricultural practices. Nature 418: 685-688.

Haslberger, A.G. 2003. GM food: The risk assessment of immune hypersensitivity reactions covers more than allergenicity. Food Agr. Environ. 1: 42-45.

Huang, J., C. Pray \& S. Roselle. 2002. Enhancing the crops to feed the poor. Nature 418: 678-684.

Kuiper, H.A., G.A. Kleter, H.P.J.M. Noteborn \& E.J. Kok. 2001. Assesment of the food safety issues related to genetically modified foods. Plant J. 27: 503-528.

Kuiper, H.A. 2003. Comparative safety assessment for biotech crops. Trends Biotechnol. 21: 439.

Nuffield Council on Bioethics. 2004. The use of genetically modified crops in developing countries. A follow-up discussion paper. Nuffield Council on Bioethics (ed.). London. p. 122.

Qaim, M., \& D. Zilberman. 2003. Yield effects of genetically modified crops in developing countries. Science 299: 900-902.

Quesada, T., J. Lobo \& A.M. Espinoza. 2002. Genetic diversity and mating system of the wild rice species Oryza Latifolia Desv. Genet. Resources Crop Evol. 49: 633-643.

Ramón, D. 2004. Presente y futuro de los alimentos transgénicos. Sistema 179-180: 31-40.

Sittenfeld, A., A. M. Espinoza, M. Muñoz, A. Zamora. 2001. Costa Rica, pp. 203-215. In G.J. Presley, \& L.R. MacIntyre (eds.). Agricultural Biotechnology: Country Case Studies-A Decade of Development. CABI, UK.

Valdez, M, R. López \& L. Jiménez. 2004a. Estado actual de la biotecnología en Costa Rica. Rev. Biol. Trop. 52: en prensa.

Valdez, M., K. Madriz \& P. Ramírez. 2004b. Establecimiento de un método de transformación genética de maíz para conferirle resistencia ulterior a enfermedades virales. Rev. Biol. Trop. 52: en prensa.

Valdez, M, I. Rodríguez \& A. Sittenfeld. 2004c. Percepción de la biotecnología en estudiantes universitarios de Costa Rica. Rev. Biol. Trop. 52: en prensa. 
Zamora-Meléndez, A., P. González \& A.M. Espinoza. 2003. Wild rice (Poaceae: Oryza) species of Costa Rica: diversity and distribution. Genet. Resources Crop Evol. 50: 855-870.

\section{REFERENCIAS DE INTERNET}

Arjona, R. 2004. Desarrollo de un Marco Nacional en Bioseguridad para Costa Rica. In A. May (ed.). Proyecto UNEP-GEF. Consultado octubre 2004, http://www.protecnet.go.cr/bioseguridad

BIO (Biotechnology Industry Organization). 2002. BIO’s Editors' and Reporters' Guide to Biotechnology. 2002-2003. Consultado agosto 2003, http:// www. bio.org.

ICSU (International Council for Science). 2003. New genetics, food and agriculture: Scientific DiscoveriesSocietal Dilemas (France: ICSU). Consultado agosto
2004, http://www.icsu.org/1_icsuinscience/INIT_G MOrep_1.html.

James C. 2003. Preview-global starus of commercialized transgenic crops. (ISAAA Briefs No 30). Consultado febrero 2004, http:/www.isaaa.org/CBT News/ press_release/briefs 30/es_b30.pdf.

MAG (Ministerio de Agricultura y Ganadería). 2004. Gerencia Técnica de Biotecnología. Consultado octubre 2004, http://www.mag.go.cr/servicios/fitosanita rio.html.

NCFAP (National Center for Food and Agricultural Policy). 2002. Plant biotechnology: current and potential impact or improving pest management in U.S. agriculture: An analysis of 40 case studies. Consultado agosto 2004, http://www.ncfap.org/40CaseStu dies.htm.

WHO (World Health Organization). 2003. 20 questions on genetically modified (GM) foods. Consultado agosto 2004. http://www.who.int/foodsafety/publica tions/biotech/20questions/en/. 